\title{
New modalities for scientific engagement in Africa - the case for computational physics
}

\author{
N. Chetty \\ Physics Department, University of Pretoria, Pretoria, 0001, South Africa
}

\section{A R T I C L E I N F O}

\section{Article history:}

Received 22 July 2010

Accepted 6 October 2010

Available online $\mathrm{xxxx}$

\section{Keywords:}

Computational physics education research

Electronic Structure Methods and

Applications

\begin{abstract}
A B S T R A C T
Computational physics as a mode of studying the mathematical and physical sciences has grown worldwide over the past two decades, but this trend is yet to fully develop in Africa. The essential ingredients are there for this to happen: increasing internet connectivity, cheaper computing resources and the widespread availability of open source and freeware. The missing ingredients centre on intellectual isolation and the low levels of quality international collaborations. Low level of funding for research from local governments remains a critical issue. This paper gives a motivation for the importance of developing computational physics at the university undergraduate level, graduate level and research levels and gives suggestions on how this may be achieved within the African context. It is argued that students develop a more intuitive feel for the mathematical and physical sciences, that they learn useful, transferable skills that make our graduates well-sought after in the industrial and commercial environments, and that such graduates are better prepared to tackle research problems at the masters and doctoral levels. At the research level, the case of the African School Series on Electronic Structure Methods and Applications (ASESMA) is presented as a new multi-national modality for engaging with African scientists. There are many novel aspects to this School series, which are discussed.
\end{abstract}

(C) 2010 Elsevier B.V. All rights reserved.

\section{Introduction}

Science has a centrally important role to play in the development of the African continent [1,2], but this point is not fully appreciated at the highest levels within African governments. Africa has been the basis of a great many sociological studies aimed at understanding the root causes of the great many challenges faced by its people. Understanding some of the problems in Africa requires a historical, cultural, sociological, economic, anthropological, etc. perspective. It is possible that we now have a better understanding of the problems and the reasons for the problems, but we are not closer to finding solutions and even further away from implementing successful solutions.

As a physicist, I have chosen to take a much simpler view: We need to get on with the job of doing quality science in Africa and to create critical mass and networking. We need to focus on both education and research. It is dangerous and even self-defeating if we aspire for lower standards of science in Africa. There is no such thing as "African science" as different from "Western science", although science in Africa or science rooted in local relevance is an entirely different matter, and must be encouraged and developed further. Science is an international endeavour and Africans must enter the knowledge arena as equal partners aiming for the high-

E-mail address: Nithaya.Chetty@up.ac.za. est possible international standards. To achieve this, we need to enhance international collaborations and encourage two-way exchanges of collaborating partners. Much is to be gained by western scientists if they make the journey to collaborate with an African partner in Africa. This way, a greater ripple effect of enhancing science is felt at the local institution.

We need to increase intra-African mobility. African scientists are notoriously more likely to board a plane to head to a western destination rather interact with other scientists on the African continent, and yet so much is to be gained by interacting and collaborating on problems of mutual interest and especially of relevance to Africa.

It is very difficult to rely on local governments to sponsor the development of science partly because of other competing priorities usually of a social nature, but perhaps more importantly because of the lack of appreciation at the highest levels of government of the importance of science as an instrument of development. It is unlikely that a single organisation or philanthropist can be found that is in a position to financially support the development of science on the entire African continent. Scientists themselves are going to have to muster a large-scale multi-national effort if we are indeed going to make the kind of impact that we want to see and that we know will make a difference. We need to find many more international sources of funding to make this happen. This will hopefully have the desired effect of making the 
local politicians and local funding agents sit up to take note of the critical importance of investing in science on the African continent.

The time for talking about doing good science is long gone - we need to be doing quality science in Africa, and this paper discusses the proposed African School on Electronic Structure Methods and Applications that is a biennial series that will rotate through a selection of African countries over the next decade. This example provides practical suggestions on how a large multi-national collaboration can engage more effectively with scientists in Africa.

Herein lies my strongest message: There is great potential for intellectual growth in Africa, and international academics and researchers have a vital role to play. There are growing opportunities for international engagement in Africa. I want to discuss this in the context of computational physics

\section{The developing ground-work for scientific research and collaborations in Africa}

Through the African Union, there is a tacit understanding of the importance of science in the development of Africa, although this declaration is yet to translate to significant improvements in the availability of funds to pursue scientific research. Countries in the Southern African Development Community (SADC) have committed themselves to spending $1 \%$ of their gross domestic product (GDP) on research and development (R\&D) - South Africa has exceeded $0.8 \%$ of its GDP on its R\&D spend, but other countries in the Community are lagging behind.

The proposed square kilometer array (SKA) is perhaps the first truly African-wide science project that has the potential of changing the dynamics of science and collaborations on the African continent in a significant way. For example, agreement has to be established at the highest levels of governments for the creation of radio frequency free zones. The potential for quality science to develop around each antenna at various rural locations on the African continent is immense. Computational expertise is only one area that must be developed to support this effort. Engineering and technical services will be essential for the upkeep of the facility. World class science will come to some of the most rural areas of Africa. The African Physical society recently endorsed the SKA as an African-wide project. Astronomy is a flagship endeavour in Southern Africa, for example the Southern African Large Telescope (SALT) and the High Energy Stereoscopic System (HESS) are largescale African observation facilities.

An exciting recent development has been the granting of $\$ 20$ million (Canadian) by the Canadian government to develop a network of institutes in Africa modelled on the very successful African Institute for Mathematical Sciences (AIMS) in Muizenberg, Cape Town, South Africa. The countries that have been identified thus far for hosting AIMS facilities are Nigeria, Senegal, Ghana and Ethiopia. During its seven years of existence, the AIMS model has demonstrated a very high level of productivity and success in the development of quality African diploma graduates in mathematics, theoretical physics and computational physics. A separate AIMS Research Centre opened in 2008 that aims to develop graduate students, postdoctoral fellows and young faculty. There is much anticipation that this success will be replicated elsewhere in Africa. As in the South African case, it is critically important that local governments also commit themselves to funding the AIMS programme.

The launching of the African Physical Society [3] (AfPS) in Senegal in January 2010 has a lot of promise for African physicists to now speak with a single voice. However, as there are challenges in managing the European Physical Society side-by-side with the other national physical societies such as the IoP and the German Physical Society (overlap=competition), so too is it quickly emerging that the AfPS needs to set an agenda and create a resource base that is distinctly different from and complementary to the national physical societies. Part of the goal of the AfPS is to help establish national physical societies where such societies do not currently exist.

There are many established and emerging pan-African initiatives that are going a long way toward helping create collaborative networks in Africa, for example the African Laser Centre (ALC), the African Laser, Atomic, Molecular and Optical Sciences network (LAM), the African Materials Research Society (AMRS) and so on. The Centre for High Performance Computing (CHPC) in Cape Town has acquired the Blue Gene supercomputer which has been designated for African collaborations. This new facility remains largely an untapped resource for African computational physicists.

The International Centre for Theoretical Physics (ICTP) through generous funding from the Italian government and UNESCO has an exemplary and long-standing engagement with Africa; the accumulative experience - including administrative experience - and networking established by the ICTP over the decades is an incredible and untapped resource for African scientists to learn more about their fellow African scientists.

Scandinavia has traditionally supported research and educational development on the African continent. France continues to have very strong educational and cultural ties with Francophone Africa. South Africa has now signed bi-national agreements with more than 30 countries including China recently, whose influence on the African continent is growing.

The Institute of Physics (IoP) [4] recently declared a strong interest in promoting physics internationally, and many initiatives are currently underway to develop physics on the African continent. For example, in December 2009, the IoP hosted a meeting in Gordon's Bay, Cape Town involving presidents of African Physical Societies to listen first-hand to ways in which the IoP can engage and assist African physicists more effectively. The meeting was jointly sponsored by the American Physical Society; the National Society for Black Physicists (USA) played a prominent role in the deliberations, and has an important role to play in the future development of physics in Africa. The South African Institute of Physics (SAIP) has recognised Africa as an important growth area for physics that must be supported and nurtured. For more than two decades now the SAIP and the Theoretical Physics Community in particular - through the Chris Engelbrecht Theoretical Physics Summer School Series, and more latterly through the National Institute for Theoretical Physics (NITheP) - have sponsored foreign African national participation at its meetings and workshops.

Legislative frameworks, policies and regulations governing scientific research and education are slowly developing in many African countries with a corresponding improvement in financial and administrative support. The groundwork is developing for scientific research and collaborations to be on a firmer footing in the coming years. We are at the stage now where more quality scientific outputs, increased impact on education and research, more graduate student production, growing innovation and an improving culture of science are all having a positive feedback into the system of science as funding regimes are responding positively in their support of science. The terrain is ripe now for African scientists in partnership with international scientists and organisations to deliver on more quality science in order that they reap the benefits of increasing investments in science in Africa. In here, computational physics has an important role to play.

\section{Why computational physics?}

Computational physics is an effective means of teaching and conducting research in the mathematical and physical sciences. It is a means of making more tangible abstract concepts, and it enhances appreciation and understanding of the subject material. In 
so doing, students learn useful transferable skills that make them more attractive in the job market. Most students come to university to secure a job rather than pursue the ideal of education and learning. This is a reality that we must recognise. Computational physics creates an opportunity for students to achieve both goals.

The growth potential of computational physics in Africa is both enormous and yet to be fully realised. At present, our universities do not have a strong culture of hands-on computing with the view to solving problems in the mathematical and physical sciences. If the general state of mathematics and physics in the country is a measure of the general state of science, engineering and technology in that country, then it is no surprise that in other scientific endeavours, such as chemistry and biology, there is even less interest and expertise in developing computer code to solve relevant problems both in research and teaching. This lack of capacity is reflected in our society-at-large where there is an over-reliance on expensive commercial software to solve problems.

Generally speaking, our students are not sufficiently computationally competent. This is clearly a short-coming in our university education system. Whenever a computational solution is sought, often the first port-of-call even amongst many fellow academics, is the internet, to try to find a package that is readily available, sometimes at an exorbitant price. The prevailing attitude is to fit the computer package to solve the problem which limits the type of problem that can be solved, how it is solved and the manner in which the results are displayed.

This exposes our general view toward technology in Africa, which is often seen as something that is imported from a foreign supplier, shipped from a far of place together with an instruction manual. We fail to see technology as being a part of a process of evolution based on our local needs with incremental improvements over a period of time. We are hardly active participants in technology, but passive recipients of foreign inputs. This malaise we must challenge and can only be achieved by providing quality human resources.

Africa has the potential to excel in computational physics, and every effort should be made to realise this potential. We must begin to entrench a culture of hands-on computing which can be encouraged by using freeware operating systems, compilers as well as graphical packages. India has been very successful in this domain, and today many Indian computer software engineers working both on the sub-continent as well as in the USA are making significant contributions to computer code development in the commercial world with enormous economic benefits. The advantages for Africa entering into this field are, of course, many-fold. Firstly, our students are schooled in practical computing, a skill that impacts on many different facets of the economy. We need software solutions to a myriad of problems that are relevant to the African continent, and we therefore need to develop an in-house capability of computer code development. There are only a few areas in life today where computers have not made a significant impact. Furthermore, there are many possibilities for jobs in the scientific, technological, educational and industrial settings, as well as careers outside the traditional scientific and technical areas, for example, the financial, insurance, governmental and business sectors. A growing number of computational experts start up their own companies focusing on computer code development, computer systems administration, web development, networking, etc. to solve specific problems needed by private commerce and industry. Africa is in dire need of young energetic graduates with a scientific and technical expertise combined with a training in practical computing.

\section{What is computational physics?}

Computational physics is a new mode of studying physics. It is sandwiched between theoretical and experimental physics, and focuses on the practical use of computers in solving physical problems. We distinguish between computational physics education research and computational physics research.

Computational physics education research has the following four distinctly different facets:

(1) The investigation of the effectiveness of using computers in the teaching of physics; the development of computational physics teaching tools. There is a technical and a sociological aspect to this type of research.

(2) The simulation of real experimental laboratories on the computer. This is useful in a developing country where real experimental equipment is scarce. For example, one can emulate the setting up of basic electric circuits using light bulbs, batteries, resistors, capacitors, etc., on the computer, and have students perform experiments on the computer in much the same way as they would do so in the laboratory.

(3) Interfacing computers in the driving of experimental equipment. For example, the protocols have to conform to the IEEE standards, and the research is distinctly experimental in nature.

(4) The development of computational paradigms to enhance the understanding of physics; the translation of current research in advanced topics to physics taught in the class room.

Computational physics research has two facets:

\section{(1) Applied computational physics which may also be referred to as Modelling}

Research in applied computational physics usually entails using some type of package or large-scale production code in the study of a physics-related problem. Usually, the basic scientific principles and theoretical model is understood, but very often there is only very little or superficial understanding of the algorithmic details, and in the case of commercial programs often there is no access to the code.

The modus operandi usually involves changing calculational parameters in 'pull-down' menus, with a 'drop, drag and off-you-go' mode of computation. The research focus here would be on the application of the computational method to real systems of interest.

This, of course, is a legitimate scientific endeavour. It is unrealistic to expect that every computational physicist should also develop his/her own production code, or have intimate knowledge of all the computational details. However, there are also problems with this approach: the exclusive focus on applied computational physics is detrimental to the long-term fundamental growth of computational sciences in Africa. This is breeding a cookbook-type of mentality that has its dangers. There currently is too much of a reliance on ready-to-go packages. In some instances, this involves expensive licensing costs. In some cases, the packages are of questionable relevance for African solutions, e.g. some of the deeplevel mining, which reaches depths in excess of $5 \mathrm{~km}$, is unique to Africa, and the seismological modelling of such systems using programs developed in Australia and Canada (countries with strong mining cultures) are not always readily transferable to the African environment. The consequences could be dire.

Basically, we do not have a strong culture of computer code development in Africa, and an exclusive mode of applied computational physics in our research and training of our graduate students is having a long-term negative impact on the production of quality graduates for research, education and for the mainstream economy. In some ways, all we are producing are computational technicians ... individuals who only know how to execute computer programs! 


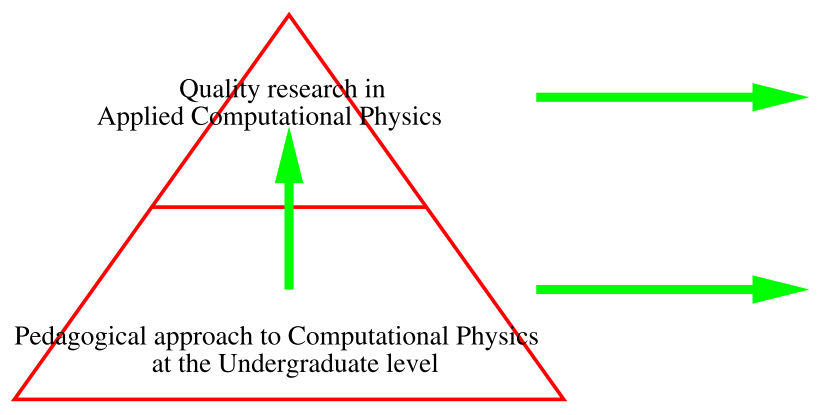

Quality research outputs on real applications

Quality graduate student production

Transferable skills to other cognate disciplines and to commerce and industry

Fig. 1. Quality undergraduate teaching of computational physics underpins quality research production in applied computational physics.

Also, there is too much reliance on Windows-based systems. It is imperative that researchers migrate to the use of open source and freeware. Linux is free and more suitable for scientific computing.

\section{(2) Fundamental computational physics}

A fundamental computational physicist pays attention to the details of all facets of the computational problem including the detailed knowledge of the underlying physical principles and the theoretical model, the development of efficient algorithms, the application of efficient numerical methods, the production of code including graphical outputs, the application to real systems of interest and the analysis of the results.

At this point in time, it is unlikely that there will be a surge in the number of individuals or groups of individuals who are involved in all these different facets of computational physics. It is very difficult to compete in the domain of algorithm and code production with very well established computational groups elsewhere in the world. It is not even wise to try to 're-invent the wheel'.

Research in applied computational physics is at this time a viable means of ensuring research productivity of the highest quality in computational physics in Africa, and this must be pursued vigourously. It is so critical, however, that this pursuit takes place in an environment that values and appreciates a fundamental approach to computational physics. For the foreseeable future, this can be accomplished by taking a more pedagogical approach to the teaching of computational physics at the undergraduate level (see Fig. 1). Much attention must be given in the coming years to this point or else we run the risk of producing computational technicians ... people who call themselves computational physicists, but who really are not computational physicists in the fundamental sense, and who are hardly able to adapt themselves to changing research, educational or commerical environments because they have no idea about how to even begin to attempt to develop a piece of production code.

\section{The undergraduate teaching of computational physics}

There are several models of quality undergraduate programmes in fundamental computational physics that one can refer to, for example the programme at Oregon State University in Corvalis, Oregon, USA that has been in operation for more than a decade. I shall briefly outline some of the key aspects that were implemented at the University of KwaZulu-Natal in Pietermaritzburg, South Africa, which has the only fully-fledged undergraduate programme in computational physics in the South Africa. The details of this programme have been reported very extensively elsewhere [5] and will therefore not be reproduced here.

It is probably not possible to introduce computational physics at the first year level because of the large number of students in- volved. It is necessary that one has an undergraduate laboratory comprising of 30-40 personal computers, and these resources will be severely taxed at the first year level.

We took the view that it was very difficult to integrate computational physics into the mainstream physics programme without impacting severely on the time available for the physics that we currently teach. Therefore, it was necessary to introduce computational physics as an additional major ... just as students could choose physics with mathematics or computer science or statistics or chemistry, etc., in the past, so do the students now have an additional option, namely computational physics. Insofar as the credits are concerned, however, computational physics constitutes half a major, which means that at the third year level, students still need to find another half a major drawn from mathematics or computer science, etc.

With the additional time devoted to computational physics teaching, it is possible to develop the subject very carefully and pedagogically, with a strong emphasis on the fundamental approaches to computing. For example, in the first semester of the second year, much attention is placed on the use of the Linux operating system, numerical methods, algorithmic development and code production using Fortran 90 and Mathematica. This means that by the time the students enter the second semester of second year, they are able to tackle interesting problems mainly drawn from computational mechanics. In the third year, the students take quantum mechanics, statistical physics, solid state physics and electromagnetism in the mainstream physics programme, and computational quantum mechanics, computational statistical physics, computational solid state physics and advanced computational statistical physics in the computational physics programme. The computational exercises and projects enhance the fundamental understanding of the core physics subjects, and the students learn useful transferable skills that enable them to find jobs, or go on to pursue high quality research degrees in theoretical and computational physics, and even in experimental physics where increasingly now computational physics is playing an important role.

\section{The graduate development of computational physics}

There are many different programmes and initiatives that are underway that aim to develop computational physics at the graduate level. The AIMS programme referred to in Section 2 above has a strong emphasis on a fundamental approach to computational physics at the graduate level. Here, I shall describe very briefly the African School on Electronic Structure Methods and Applications (ASESMA), which is a biennial school series that began in 2010 in Cape Town, South Africa, and will continue for the next decade moving through different locations in Africa, and is based principally on applied computational physics, namely the quantum mechanical modelling of real materials. 


\subsection{The African School on Electronic Structure Methods and Applications (ASESMA)}

Developments of new theoretical methods, algorithms and widely available codes have brought a new era in computational materials science [6]. This is a field of active research today because it is making possible new understanding and abilities to predict properties of materials. Because of the advent of faster, cheaper computers, new algorithms, and the availability of open source software, such as the Quantum Espresso Codes [7], there is a world-wide community of scientists developing new methods and carrying out research on important materials problems. With improving networking infrastructure, it has now become viable for African researchers to join in on this exciting international research effort.

The methods involve density functional theory [8], which makes possible calculation of materials properties from the fundamental laws with no parameters, and the methods pioneered by Car and Parrinello [9], which make it feasible to move atoms to determine structures and bonding of molecules and solids. It is now possible to make quantitative calculations for complex systems such as nanotubes with defects, transition metal alloys with light elements where it is essential to move the atoms to form strong metal-carbon, metal-nitrogen, etc., bonds that are crucial for the strength of materials. Mechanical strength and elastic properties, along with electronic, magnetic and optical properties of materials can now be readily computed, all with the same theoretical methods and the same computational codes. This opens a vast terrain for extensive studies of materials for their scientific and technological value.

The field is evolving rapidly and the School will develop new themes for future Schools in order use the most advanced methods and to focus upon the issues in materials science most relevant for Africa.

The School series is designed to ensure continuity over the next ten years, to build critical mass and to create a legacy of computational materials science in Africa. A major objective of this School series is to enhance scientific collaboration and networking in Africa. A special effort is being made to seek African women participants. The School series is managed by an International Advisory Panel (IAP) and the practicalities arranged by a Local Organising Committee (LOC).

\subsection{School programme}

The School is held over two weeks, and each School is focused on a particular theme, e.g. hard materials, optical properties, defects, surfaces, etc. The first four days involve basic lectures, including tutorial work, on topics in physics and computational physics that are relevant to the School theme. International experts are invited to lead discussions on current challenges in solid state physics and material science. For the following ten days, the School focuses on hands-on computational work. For these purposes, the hosting institution provides a networked computer laboratory comprising about 40 computers running Linux with technical support. The School programme is open to different computational packages, and particular lecturers are free to advance their own computational methods. However, in the African context, freeware in encouraged. The School is open to lecturers from the many different communities within the electronic structure world. The School caters for approximately 40 students, drawn from young faculty and graduate students from across Africa.

\subsection{Applied computational physics}

The School series is focused on applied computational physics in the following two key respects:
(1) Participants learn aspects related to hands-on computing within an open source environment. The skills acquired are transferable to other disciplines and in areas of activity outside academia, and in the mainstream economy.

(2) The applications of the computational methods are to topics in material science, which impacts on the fundamental understanding of materials, and which has the potential of adding economic value to the vast reserves of minerals and materials mined in Africa.

\subsection{Mentorships, collaborations and networking}

Participants have the opportunity to get coupled with international mentors to continue with collaborations and mutual visits and by way of pre-School and post-School activities during the period of time between Schools. This ensures research productivity and continuity. Collaborations are also facilitated amongst African researchers, and intra-African mobility is essential. A database of African students and researchers working in the field of Electronic Structure computations is being setup to enhance networking within Africa and to help seek out collaborating partners outside Africa and to facilitate funding applications.

\subsection{The 2010 School}

ASESMA was co-sponsored by the International Union for Pure and Applied Physics (IUPAP) Commissions on Physics for Development (C13), and Computational Physics (C20). The Commission on Physics Education (C14) and the Commission on Structure and Dynamics of Condensed Matter (C10) have endorsed the School series. The International Centre for Theoretical Physics (ICTP), National Institute for Theoretical Physics (NITheP), International Center for Materials Research (ICMR), American Physical Society (APS), The Centre of Excellence in Strong Materials (CoESM), The National Research Foundation and Department of Science and Technology (NRF/DST), The Materials Computation Center at the University of Illinois (MCC) and Democratis have been co-sponsors of ASESMA2010. The School was organised by the ICTP and the SAIP, and hosted at the African Institute for Mathematical Sciences in Muizenberg, Cape Town. The funds raised were in excess of 60000 Euros.

There were more than 150 applicants drawn from across the African continent; following strict selection criteria, 46 participants were offered places at the School, and 40 participants attended the School. The main target group was advanced graduate students and young faculty. Air travel, and full board and lodging were provided for the participants in the vast majority of cases.

\section{Final comments}

The expectation is that ASESMA will result in increasing international collaborations including intra-African collaborations, more investment in local computational resources, access by African scientists to international computational resources for example the Blue Gene supercomputer at the Centre for High Performance Computing (CHPC) in Cape Town, more optimal use of computational resources, more migration of computational graduates to other cognate disciplines including commerce and industry, more quality research outputs including publications, graduate student production and presentations at international conferences.

Positive feedback of this nature will continue to pressure local governments to invest more in science development in Africa.

The International Union for Pure and Applied Physics (IUPAP) played a crucial role in bridging the divide between East and West 
during the cold war years. Today, the IUPAP has positioned itself very clearly in a developmental role as adjudged by public statements made by recent presidents, as well as by resolutions passed at the General Assembly that have a distinct aim of promoting physics in under-represented communities, including women in physics.

South Africa was a founding member of the IUPAP, and several African countries have joined the IUPAP over the past five years. There is an expectation that membership of the IUPAP derives benefits for African physics. It is therefore appropriate that the annual Conference on Computational Physics (CCP) nurtures the development of computational physics research and education in the developing world. The challenge to future organisers of CCP is to include participation from the developing world.

\section{Acknowledgements}

The author is grateful to Robert Lindebaum, the late Clive Graham, and Vincent Couling who all helped develop the undergraduate programme in computational physics at the University of KwaZulu-Natal. The author acknowledges useful discussions with
Richard Martin, Sandro Scandolo, Jim Gubernatis and Kennedy Reed.

\section{References}

[1] N. Chetty, S. Connell, A.C. Bawa, Physics for development, Nature Phys. 3 (11) (November 2007).

[2] N. Chetty, A.C. Bawa, Developing computational mathematics in Africa, in: G. Sica (Ed.), What Mathematics from Africa? Polimetrica, ISBN 88-7699-023-2, 2005.

[3] S. Connell, Launch of the African Physical Society, http://www.saip.org.za/ PhysicsComment; http://www.africanphysicalsociety.org.

[4] B. Masara, IoP organises meeting with African Physical Societies, http://www. saip.org.za/PhysicsComment.

[5] N. Chetty, F. Petruccione, R.J. Lindebaum, Computational physics in South Africa, S. Afr. J. Sci. 101 (2005).

[6] R.M. Martin, Electronic Structure: Basic Theory and Practical Methods, Cambridge Univ. Press, 2004

[7] http://www.quantumespresso.org.

[8] P. Hohenberg, W. Kohn, Inhomogeneous electron gas, Phys. Rev. B 136 (1964) 864-871;

W. Kohn, L.J. Sham, Self-consistent equations including exchange and correlation effects, Phys. Rev. A 140 (1965) 1133-1138.

[9] R. Car, M. Parrinello, Unified approach for molecular dynamics and density functional theory, Phys. Rev. Lett. 55 (1985) 2471-2474. 